\title{
SOME EXAMPLES OF SPHERE BUNDLES OVER SPHERES WHICH ARE LOOP SPACES $\bmod p$
}

\author{
BY JOHN EWING
}

Communicated by Morton Curtis, March 26, 1974

\begin{abstract}
In this note we give sufficient conditions that certain sphere bundles over spheres, denoted $B_{n}(p)$, are of the homotopy type of loop spaces $\bmod p$ for $p$ an odd prime. The method is to construct a classifying space for the $p$-profinite completion of $B_{n}(p)$ by collapsing an Eilenberg-Mac Lane space by the action of a certain finite group.
\end{abstract}

We say that a space $X$ has some property $\bmod p$ if the localization of $X$ at $p$ has the property. The problem of determining which spheres are of the homotopy type of loop spaces $\bmod p$ has been completely solved by Sullivan [9]. It is therefore natural to ask which sphere bundles over spheres are of the homotopy type of loop spaces mod $p$. In this regard, results of Curtis [2] and Stasheff [7] concerning the question of which sphere bundles over spheres are $H$-spaces $\bmod p$ give some negative information. Moreover, in a recent paper [3] we investigated a certain class of sphere bundles over spheres and gave necessary conditions for them to be of the homotopy type of a loop space $\bmod p$ for $p$ an odd prime. In this note we prove that certain of these bundles satisfying the conditions of [3] are of the homotopy type of a loop space $\bmod p$ and answer a question posed in [8].

For $p$ an odd prime and $n$ a positive integer, the space $B_{n}(p)$ is an $S^{2 n+1}$-bundle over $S^{2 n+1+2(p-1)}$ classified by the generator of the $p$-primary part of $\pi_{2 n+2(p-1)}\left(S^{2 n+1}\right)$. From [5] we have that $H^{*}\left(B_{n}(p) ; Z \mid p\right)$ is an exterior algebra on generators $x$ and $y$, where $\operatorname{deg} x=2 n+1, \operatorname{deg} y=2 n+$ $2 p-1$ and $\mathscr{P}^{1} x=y$. Although few of the $B_{n}(p)$ are of the homotopy type of a loop space $\bmod p$ (see [3]), we have the following exceptions.

THEOREM 1. The space $B_{n}(p)$ is of the homotopy type of a loop space $\bmod p$ if $n$ and $p$ satisfy any of the following conditions:

(i) $n=1 ; p=$ any odd prime,

(ii) $n=p-2 ; p=$ any odd prime,

(iii) $n=7 ; p=17$,

(iv) $n=5 ; p=19$,

(v) $n=19 ; p=41$.

AMS (MOS) subject classifications (1970). Primary 55F25, 55F35. 
REMARK. Two cases of Theorem 1 follow immediately from [5]: namely $B_{1}(3)$ is of the homotopy type of $\mathrm{Sp}(2) \bmod 3$, and $B_{1}(5)$ is of the homotopy type of $G_{2} \bmod 5$.

In order to prove Theorem 1, we must introduce the $p$-profinite completion of a space as defined in [9]. For precise statements of some of the pertinent theorems, see [4]. If $X$ is a space, let $\hat{X}_{p}$ denote the $p$-profinite completion of $X$; for notational convenience we make the following conventions:

$$
\begin{aligned}
& L_{n}(p)=\text { localization of } B_{n}(p) \text { at } p . \\
& C_{n}(p)=p \text {-profinite completion of } B_{n}(p) .
\end{aligned}
$$

THEOREM 2. The space $C_{n}(p)$ is of the homotopy type of a loop space if $n$ and $p$ satisfy any one of the conditions of Theorem 1 .

Proof of Theorem 1. Theorem 1 now follows from Theorem 2 using techniques of [9]. Suppose $C_{n}(p)$ is a loop space, and let $B C_{n}(p)$ denote the classifying space. Let $W$ denote the homotopy pull-back in the following diagram:

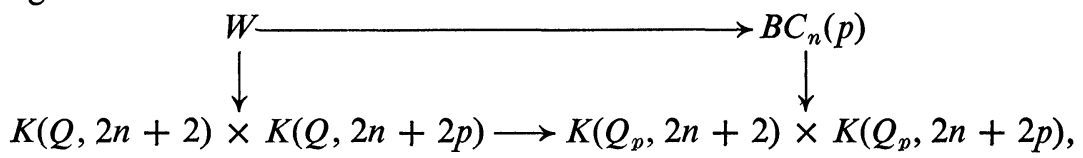

where $Q_{p}$ denotes the $p$-adic numbers. Looping the diagram we conclude that $L_{n}(p) \simeq \Omega W$. Q.E.D.

The proof of Theorem 2 is somewhat involved and so we outline the procedure. Given $n$ and $p$ satisfying one of the conditions, we construct two $p$-profinitely complete spaces, $A$ and $X$, together with a map $i$ : $A \rightarrow X$. We show $\Omega A \simeq \hat{S}_{p}^{2 n+1}, H^{*}(\Omega X ; Z \mid p) \approx H^{*}\left(B_{n}(p) ; Z \mid p\right)$ as modules over the Steenrod algebra, and $(\Omega i)^{*}: H^{2 n+1}(\Omega X ; Z / p) \rightarrow H^{2 n+1}(\Omega A ; Z / p)$ is an isomorphism. We conclude that there is a map $f: S^{2 n+1} \rightarrow \Omega X$ such that $f^{*}: H^{2 n+1}(\Omega X ; Z \mid p) \rightarrow H^{2 n+1}\left(S^{2 n+1} ; Z / p\right)$ is an isomorphism. From [5] we have the following cell structure for $B_{n}(p)$ :

$$
B_{n}(p) \cong S^{2 n+1} \cup_{\alpha} e^{2 n+2 p-1} \cup e^{4 n+2 p} .
$$

Since $\mathscr{P}^{1}$ is nontrivial on $H^{*}(\Omega X ; Z / p)$, we conclude $f \propto$ is null homotopic. Therefore, by proving $\pi_{4 n+2 p-1}(\Omega X)$ is trivial, we have shown that $f$ extends to a map $f: B_{n}(p) \rightarrow \Omega X$. By functoriality of $\mathscr{P}^{1}$ and cup products, this extension induces an isomorphism of $\bmod p$ cohomology. From [9] or [4] we have that $C_{n}(p) \simeq \Omega X$.

In this note we give details of the construction only in the case $n=1$. The remaining cases are similar and details can be found in [4]. Let $\boldsymbol{Z}_{\boldsymbol{p}}$ denote the $p$-adic integers, and let $\theta$ be a primitive $(p+1)$ st root of unity. 
It is easily verified that $\theta+\theta^{-1}$ and $\left(\theta-\theta^{-1}\right)^{2}$ are in $\hat{Z}_{p}$. Let $D_{p+1}$ denote the dihedral group of order $2(p+1)$ in $\mathrm{GL}\left(2, \hat{Z}_{p}\right)$ generated by

$$
\frac{1}{2}\left(\begin{array}{cc}
\theta+\theta^{-1} & \left(\theta-\theta^{-1}\right)^{2} \\
1 & \theta+\theta^{-1}
\end{array}\right) \text { and }\left(\begin{array}{rr}
-1 & 0 \\
0 & 1
\end{array}\right) \text {. }
$$

Let $C_{2}$ denote the cyclic group of order 2 in $D_{p+1}$ generated by the second element above. Let $j: \hat{Z}_{p} \rightarrow \hat{Z}_{p} \times \hat{Z}_{p}$ be inclusion into the first factor. We proceed as in [1]. The natural actions of $C_{2}$ and $D_{p+1}$ on $\hat{Z}_{p}$ and $\hat{Z}_{p} \times \hat{Z}_{p}$ induce actions on the Eilenberg-Mac Lane spaces $K\left(\hat{Z}_{p}, 2\right)$ and $K\left(\hat{Z}_{p} \times \hat{Z}_{p}, 2\right)$. Let $E D_{p+1}$ be an acyclic complex on which $D_{p+1}$ acts freely, and let $C_{2}$ and $D_{p+1}$ act on $K\left(\hat{Z}_{p}, 2\right) \times E D_{p+1}$ and $K\left(\hat{Z}_{p} \times \hat{Z}_{p}, 2\right) \times E D_{p+1}$ by diagonal actions. Let $A$ and $X$ denote the $p$-profinite completions of the respective orbit spaces. Denote by $i: A \rightarrow X$ the map induced by $j$. From [1] we can conclude:

$$
\begin{aligned}
H^{*}(A ; Z / p) & \approx Z \mid p[x], \quad \operatorname{deg} x=4 ; \\
H^{*}(X ; Z / p) & \approx Z \mid p[u, v], \quad \operatorname{deg} u=4, \quad \operatorname{deg} v=2 p+2 ; \\
i^{*}(u) & =x \quad \text { and } \mathscr{P}^{1} u=v \quad \text { (see [2]). }
\end{aligned}
$$

If we consider loop spaces we have correspondingly:

$$
\begin{aligned}
& H^{*}(\Omega A ; Z \mid p) \approx E(\bar{x}), \quad \operatorname{deg} \bar{x}=3 ; \\
& H^{*}(\Omega X ; Z \mid p) \approx E(\bar{u}, \bar{v}), \quad \operatorname{deg} \bar{u}=3, \quad \operatorname{deg} \bar{v}=2 p+1 ; \\
& (\Omega i)^{*}(\bar{u})=\bar{v} \quad \text { and } \quad \mathscr{P}^{1} \bar{u}=\bar{v} .
\end{aligned}
$$

Since $\Omega A$ is a simply-connected, $p$-profinitely complete space, we have $\Omega A \simeq \hat{S}_{p}^{3}$. Therefore we get a map $f: S^{3} \rightarrow \Omega X$ such that $f^{*}(\bar{u}) \neq 0$. From the above remarks, to extend $f$ to $B_{1}(p)$ we need only show $\pi_{2 p+3}(\Omega X)=0$.

Consider the diagram:

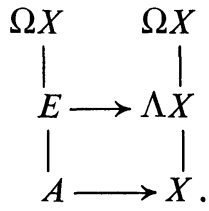

The pull-back $E$ is a simply-connected p-profinitely complete space. Moreover, we can compute $H^{*}(E ; Z / p)$ from the Eilenberg-Moore spectral sequence, which collapses [6] and gives $H^{*}(E ; Z / p)$ as an exterior algebra on a generator of degree $2 p+1$. We conclude $E \simeq S_{p}^{2 p+1}$. Since $\pi_{2 p+4}(A) \approx \pi_{2 p+3}\left(S_{p}^{3}\right)=0$ and $\pi_{2 p+3}\left(S_{p}^{2 p+1}\right)=0$, we have $\pi_{2 p+3}(\Omega X)=0$.

Therefore, $f$ extends to a map $f: B_{1}(p) \rightarrow \Omega X$ which induces an isomorphism on $\bmod p$ cohomology. We conclude that $C_{1}(p) \simeq \Omega X$. 


\section{REFERENCES}

1. A. Clark and J. Ewing, The realization of polynomial algebras as cohomology rings, Pacific J. Math. (to appear).

2. M. Curtis, $H$-spaces mod p. II, $H$-spaces (Actes Réunion, Neuchâtel, 1970), Lecture Notes in Math., vol. 196, Springer-Verlag, Berlin, 1971, pp. 11-19. MR 44 \#1028.

3. J. Ewing, Sphere bundles over spheres as loop spaces $\bmod p$, Duke Math. J. 40 (1973), 157-162.

4. - p-profinite analogues of the classical Lie groups (in preparation).

5. M. Mimura and H. Toda, Cohomology operations and homotopy of compact Lie groups. I, Topology 9 (1970), 317-336. MR 42 \#1144.

6. L. Smith, Homological algebra and the Eilenberg-Moore spectral sequence, Trans. Amer. Math. Soc. 129 (1967), 58-93. MR 35 \#7337.

7. J. Stasheff, Sphere bundles over spheres as $H$-spaces mod $p>2$, Sympos. on Algebraic Topology, Lecture Notes in Math., vol. 249, Springer-Verlag, Berlin and New York, 1971.

8. - Infinite loop spaces-a historical survey, $\mathrm{H}$-spaces (Actes Réunion Neuchâtel, 1970), Lecture Notes in Math., vol. 196, Springer-Verlag, Berlin and New York, 1971, pp. 43-53. MR 43 \#6911.

9. D. Sullivan, Geometric topology, M.I.T. Notes, Cambridge, Mass.

Department of Mathematics, Dartmouth College, Hanover, New Hampshire 03755

Current address: Department of Mathematics, Indiana University, Bloomington, Indiana 47401 